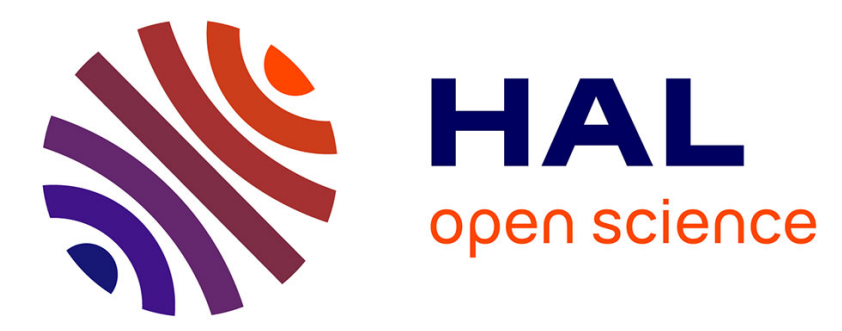

\title{
La reconquête du marché intérieur et la relance économique: esquisse d'une stratégie André Grjebine
}

\section{To cite this version:}

André Grjebine. La reconquête du marché intérieur et la relance économique : esquisse d'une stratégie. Revue Economique, 1982, 33 (6), pp.1115-1140. 10.3406/reco.1982.408700 . hal-01009224

\section{HAL Id: hal-01009224 \\ https://hal-sciencespo.archives-ouvertes.fr/hal-01009224}

Submitted on 17 Jun 2014

HAL is a multi-disciplinary open access archive for the deposit and dissemination of scientific research documents, whether they are published or not. The documents may come from teaching and research institutions in France or abroad, or from public or private research centers.
L'archive ouverte pluridisciplinaire HAL, est destinée au dépôt et à la diffusion de documents scientifiques de niveau recherche, publiés ou non, émanant des établissements d'enseignement et de recherche français ou étrangers, des laboratoires publics ou privés.

\section{(ㅇ)(1) $\$$}

Distributed under a Creative Commons Attribution - NonCommercial - NoDerivatives| 4.0 


\title{
La reconquête du marché intérieur et la relance économique :
}

\section{esquisse d'une stratégie}

In: Revue économique. Volume 33, n6, 1982. pp. 1115-1140.

\begin{abstract}
Résumé
La crise actuelle s'inscrit à la croisée de deux évolutions pernicieuses : un endettement de plus en plus fort des agents économiques et une contrainte extérieure de plus en plus pesante. D'où la stratégie préconisée dans cet article : 1) orienter la relance de l'économie vers une réduction de notre propension à importer, en premier lieu par un développement accéléré de nouvelles sources d'énergie ; 2 ) modifier les contreparties de la masse monétaire en remplaçant progressivement la création de monnaie par le crédit (et donc par endettement des agents économiques) par une création monétaire par l'Etat. Celle-ci permettra simultanément de financer la relance autocentrée suggérée et de désendetter les entreprises.
\end{abstract}

\section{Abstract}

PROPOSALF TO RECONQUER THE INTERNAL MARKET AND TO STEP UP THE ECONOME

André Grjebine

The present crisis is aggravated by two convergent trends : the growing indebted-ness of economic agents and increasingly heavy external constraints. This explains the strategy proposed in the following article : 1 . to stimulate economic growth by braking the tendency to import and 2. to change the assets of the monetary mass by gradually replacing the creation of money, now done by credit and there-fore by indebtedness of économie agents, by State money creation. The latter has the double advantage of financing autonomous economic growth and freeing enterprises from their debt burden.

Citer ce document / Cite this document :

Grjebine André. La reconquête du marché intérieur et la relance économique : esquisse d'une stratégie. In: Revue économique. Volume 33, n6, 1982. pp. 1115-1140.

http://www.persee.fr/web/revues/home/prescript/article/reco_0035-2764_1982_num_33_6_408700 


\title{
LA RECONQUETE DU MARCHE INTERIEUR ET LA RELANCE ECONOMIQUE : ESQUISSE D'UNE STRATEGIE
}

\begin{abstract}
Eule une relance durable et de grande ampleur peut renverser l'évoIution actuelle du marché du travail et réduire progressivement $\checkmark$ le chômage. Mais la contrainte extérieure qui pèse sur notre économie condamne toute relance «classique» par la consommation à déboucher sur un déficit extérieur croissant qui rendra rapidement inévitable le retour à l'austérité. Une relance forte et durable n'est donc envisageable que si elle est orientée de manière à réduire notre propension à importer et à éviter des retombées inflationnistes qui ne manqueraient pas de porter atteinte à la compétitivité de notre industrie et, ce faisant, d'accroître le déficit commercial.
\end{abstract}

En fait, si les gouvernements occidentaux paraissent désarmés face à la crise qu'ils doivent affronter, c'est qu'elle diffère considérablemènt de celles qui l'ont précédée. Ces dẹnières se caractérisaient généralement par un mal qui pouvait atteindre un extrême degré de gravité mais qui était prééminent à un moment donné : récession profonde ou inflation galopante. Il s'agissait donc, selon le dilemme bien connu, de combattre la récession en acceptant une certaine dose d'inflation ou de lutter contre cette dernière, quitte à accepter un ralentissement de l'expansion. La situation actuelle n'offre pas un tel choix puisque les trois maux - récession, inflation, déficit extérieur - coexistent et paraissent même étroitement associés. On ne saurait donc combattre un seul de ces maux, non seulement sans aggraver les deux autres, mais même sans que cette aggravation ne conduise en fin de compte à une détérioration globale de la situation. L'échec patent de la politique menée pendant cinquante-six mois par M. Raymond Barre apparaît comme une illustration flagrante de cet état de fait. Est-ce à dire que la situation est inextricable? 


\section{Revue économique}

Certainement pas. On peut même trouver dans le passé des situations qui n'étaient pas sans analogie avec celles que nous connaissons actuellement et qui ont été beaucoup mieux surmontées que les récessions traditionnelles. Par exemple, à la fin des années 1930, alors qu'une politique de relance trop timide ne parvenait pas à extirper le chômage qui sévissait encore aux Etats-Unis, la politique de réarmement accéléré entreprise dans le cadre de la loi prêt-bail a permis simultanément de vaincre le chômage, de mettre sur pied en quelques mois une industrie d'armement extrêmement puissante, enfin de désendetter les agents économiques en remplaçant partiellement la création monétaire par le système bancaire par une émission monétaire par la voie d'un déficit budgétaire massif - et nous verrons ultérieurement que l'endettement excessif des agents économiques est précisément à l'heure actuelle une des causes majeures de l'inflation. Quelques années plus tôt, inspirée par la pensée d'Ernst Wagmann, l'Allemagne avait pratiqué une politique similaire, de manière encore plus systématique, et avait obtenu des résultats spectaculaires. Il suffit de remplacer « réarmement 》 par « reconquête de l'indépendance économique» pour voir à quel point notre situation est comparable à celle qu'a affrontée avec succès Franklin D. Roosevelt. Simplement, les armes de l'indépendance ont partiellement changé de nature... mais la politique économique susceptible d'assurer cette indépendance est toujours la même.

Cette étude vise à montrer que les trois objectifs - relancer l'économie pour réduire le chômage, extirper les racines mêmes du déficit commercial, atténuer l'inflation - non seulement ne sont pas incompatibles, mais sont au contraire complémentaires et peuvent être atteints simultanément par une politique économique adaptée.

\section{LA RECONQUETE DU MARCHE INTERIEUR CONDITION D'UNE RELANCE DURABLE}

Faute d'une réduction importante de notre propension à importer c'est-à-dire de l'accroissement des importations provoqué par une expansion du PIB - toute reprise de l'activité économique entraînera immanquablement une augmentation parallèle, voir supérieure, des importations. En revanche, une croissance de même ampleur des exportations est tout à fait improbable. En effet, celles-ci sont, avant tout, déterminées par l'évolution de la demande extérieure. Or cette der- 
nière restera déprimée aussi longtemps que la plupart de nos principaux partenaires ne seront pas sortis de la récession. L'observation des politiques adoptées dans ces pays suggère qu'ils ne sont pas prêts de le faire.

Dans un premier temps, cette relance orientée vers la reconquête du marché intérieur provoquera un accroissement des importations. Mais, alors que dans l'hypothèse d'une relance non orientée, l'endettement extérieur ne servira qu'à financer une consommation de produits importés qui ne cessera de croître tant que cette politique n'aura pas été abandonnée, dans l'hypothèse d'une relance autocentrée, l'endettement extérieur financera des investissements qui permettront d'éliminer progressivement les racines mêmes du déficit extérieur.

$\mathrm{Au}$ fur et à mesure que la reconquête du marché intérieur progressera, la contrainte extérieure va se desserrer et la propension à importer se réduire. La politique de relance pourra donc non seulement être durablement poursuivie, mais elle pourra même être renforcée. De plus, son impact sera d'autant plus fort que la demande s'adressant aux producteurs nationaux ne va pas seulement augmenter en raison des effets induits du déficit budgétaire mais aussi parce qu'une partie croissante du pouvoir d'achat actuellement consacrée aux importations sera "ramenée 》 vers l'achat de produits français.

Après avoir mis en évidence la supériorité d'une relance autocentrée par rapport à une relance non sélective à l'aide de schémas chiffrés, nous examinerons ce qui devrait constituer le point d'application prioritaire d'une telle relance autocentrée à savoir la réduction accélérée de notre dépendance énergétique. 
Schéma simplifié d'une relance non sélective

Hypothèses : relance par un déficit budgétaire de 100 [renouvelé la deuxième année]; propension à importer : 20\%; gains de productivité : 2\% par an.

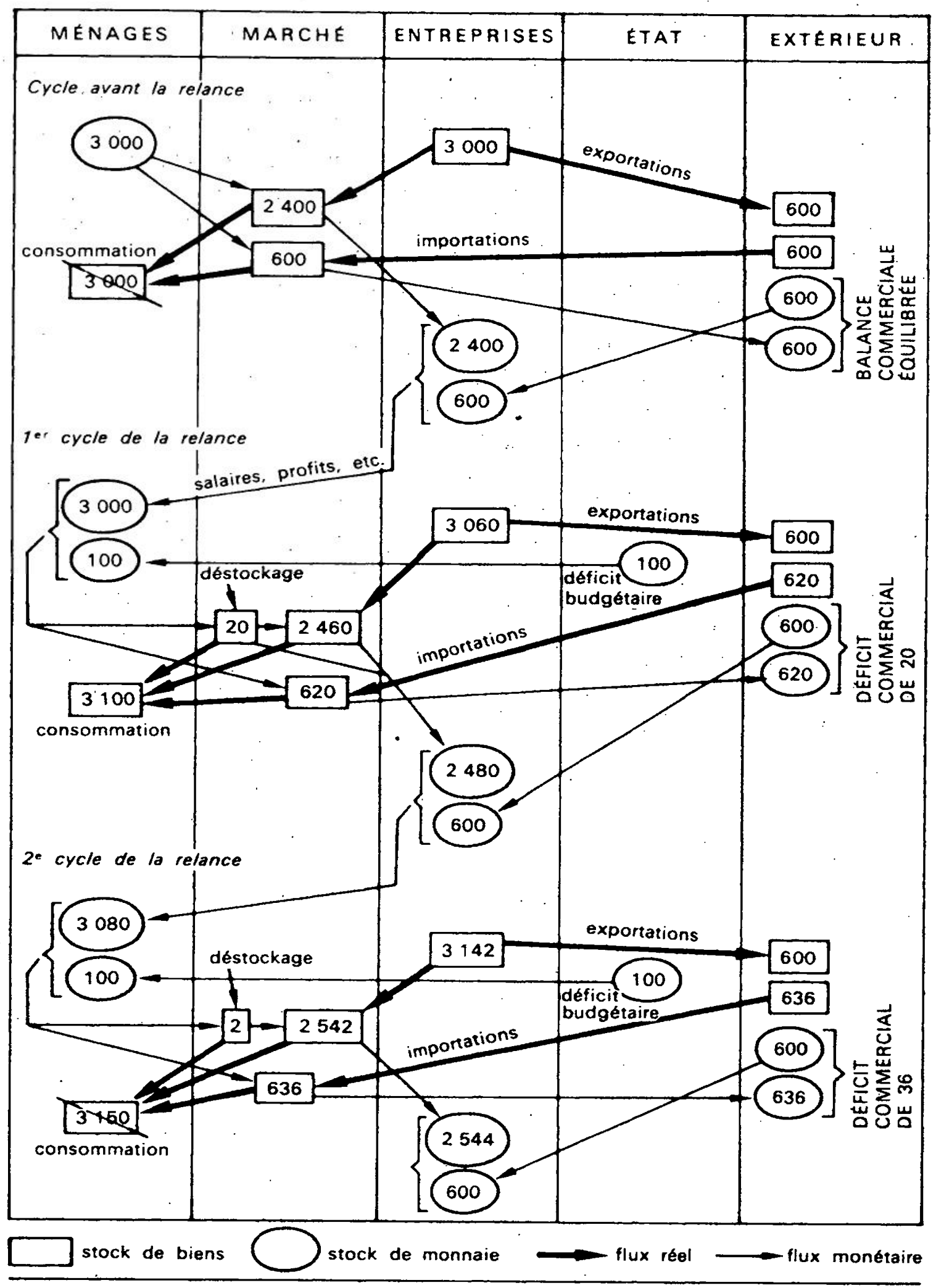

Source : André Grjebine, La nouvelle économie internationale, 2e éd., Paris, PUF, 1982. p. 317. 
Schéma d'une relance autocentrée

Réduction de la dépendance énergétique

Hypothèses : relance par un déficit budgétaire de 100 [renouvelé la deuxième année] ; propension à importer : $20 \%$ au début du processus, régresse ensuite au fur et à mesure que la production nationale d'énergie augmente; gains de productivité : $2 \%$ par an; un investissement de 100 permet une augmentation des capacités de production d'énergie de 15.

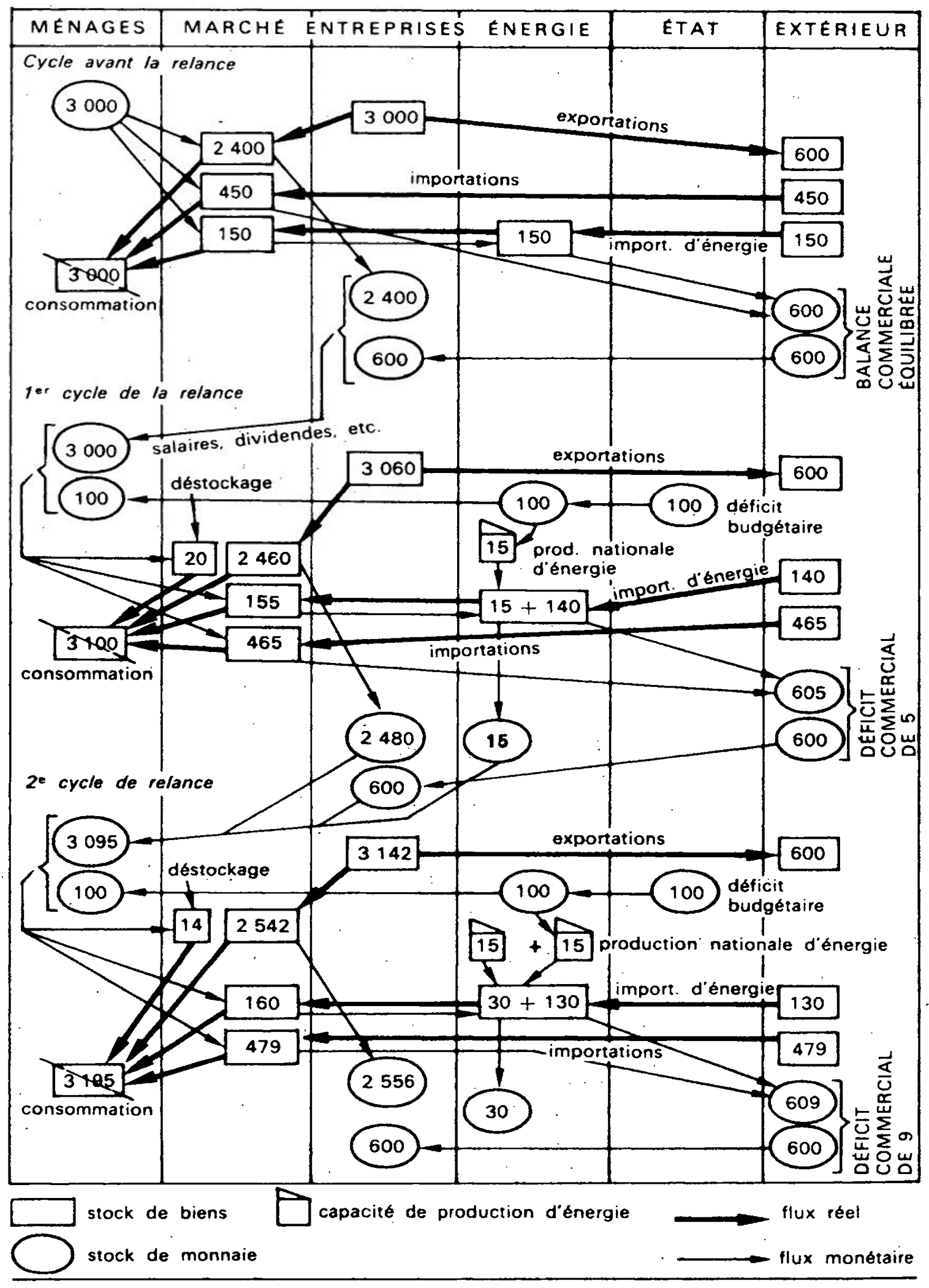

Source: André Grjebine, La nouvelle économie internationale, 2e éd., Paris, PUF, 1982, p. 318. 
Bilans compatrés des deux types de relance

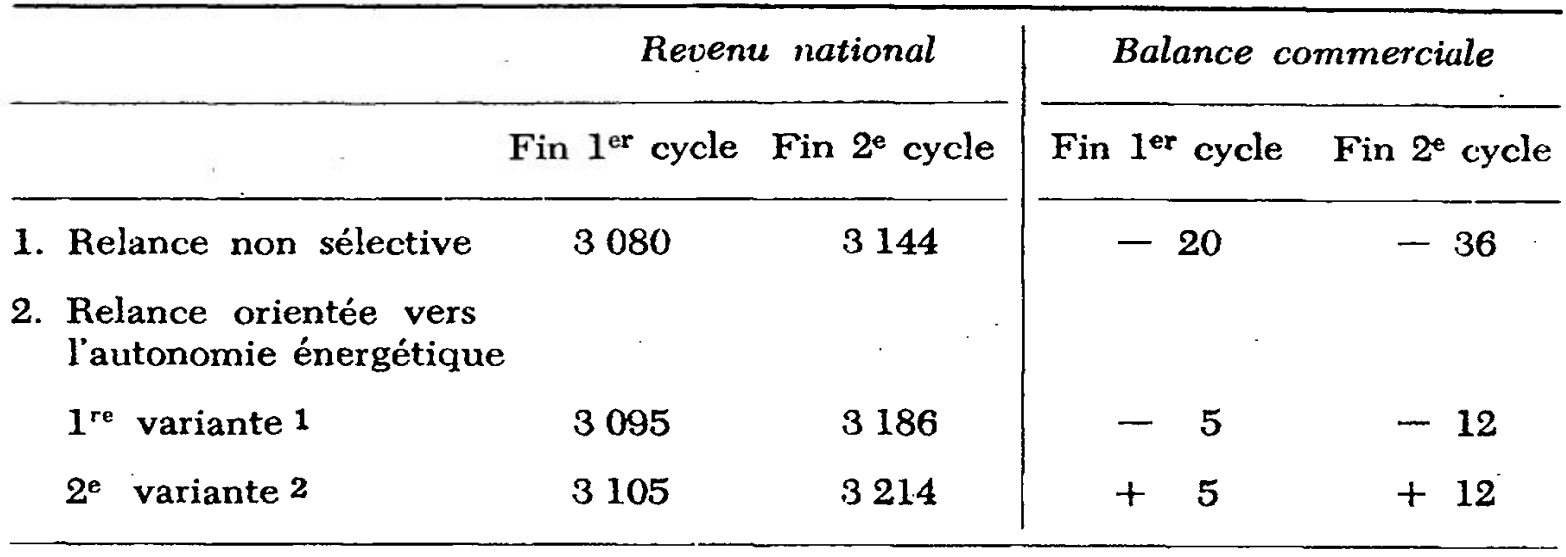

1. Un investissement de 100 permet une augmentation des capacités de production d'énergie de 15 (variante représentée par le second schéma).

2. Un investissement de 100 permet une augmentation des capacités de production d'énergie de 25 (variante non représentée schématiquement).

\section{Conclusion}

Une relance orientée vers la recherche de l'autonomie énergétique apparaît très supérieure à une relance non orientée aussi bien du point de vue de la croissance du revenu national que de celui de la balance commerciale.

\section{Remarques}

1. L'impact positif d'une relance orientée vers l'autonomie énergétique sera d'autant plus grand que :

- le prix des produits importés (pétrole) sera plus élevé et donc que l'économie réalisée grâce au développement de capacités de production nationale sera plus forte ;

- la rentabilité de l'investissement sera plus forte. L'observation des deux variantes envisagées montre que si l'investissement de 100 permet une augmentation des capacités de production d'énergie nationale supérieure aux importations provoquées par un accroissement de 100 du revenu national, non seulement cette relance n'entraînera pas de déficit commercial, mais au contraire le solde commercial sera positif et ne cessera de s'améliorer. Il va sans dire que la rentabilité des investissements énergétiques dépend des technologies adoptées. Le rapport de la cornmission Prospective de la consommation d'énergie à long terme du VIII ${ }^{\mathbf{e}}$ Plan évalue le coût par tep économisée à $12000 \mathrm{~F}$ dans le tertiaire et le résidentiel, à $10000 \mathrm{~F}$ dans les transports et à $6000 \mathrm{~F}$ dans l'industrie. En tenant compte de ces estimations d'une part, du prix du pétrole et du taux de change dollar/ franc en septembre 1981 d'autre part, on arrive à la conclusion qu'un investissement de 10 milliards de francs permettra d'économiser 3 milliards dans l'industrie, ou 1,75 milliard dans les transports, ou 1,5 milliard dans le résidentiel et le tertiaire. Il apparaît cependant que le recours à des technologies combinées (centrales produisant simultanément de l'électricité et de l'eau chaude pour le chauffage des villes, etc.) permettrait d'accélérer les investissements et d'en accroître sensiblement la rentabilité. 
2. Dans les processus simplifiés que nous avons analysés, nous n'avons pas tenu compte de deux phénomènes jouant en sens inverse l'un de l'autre mais pas forcément avec la même ampleur :

- une partie du matériel permettant d'accroître la production nationale d'énergie (ou les économies d'énergie) devra être importée, au moins dans un premier temps, et l'impact favorable de cette politique sur la balance commerciale en sera réduit d'autant. Naturellement, au fur et à mesure que les investissements seront réalisés, seuls les effets positifs inhérents à la réduction des importations d'énergie demeureront. De plus, une politique globale de reconquête du marché intérieur comme celle qui est envisagée ici devrait s'attacher en priorité à réduire notre dépendance dans les équipements nécessaires pour mener à bien la politique énergétique. Ces importations induites par une relance orientée vers l'autonomie énergétique devraient donc s'atténuer rapidement;

- les économies d'énergie réalisées en particulier dans l'industrie améliorent la compétitivité des entreprises qui peuvent, de ce fait, gagner de nouveaux marchés et accroître leurs exportations.

\section{LA PRINCIPALE ARME DE LA RECONQUETE \\ DU MARCHE INTERIEUR N'EST PAS LE PROTECTIONNISME MAIS LA DYNAMISATION DU TISSU INDUSTRIEL}

La reconquête du marché intérieur ne vise pas à l'autarcie. Il ne s'agit pas de veiller à ce que la consommation nationale soit entièrement satisfaite par une production nationale, ce qui ne pourrait se faire qu'au préjudice de la liberté de choix des consommateurs et favoriseraiit la sclérose des entreprises qui bénéficient de «marchés garantis ». A moins de se lancer dans une planification complète et fine dont on observe ailleurs les méfaits bureaucratiques, on ne saurait non plus envisager une politique subventionnant le développement de toutes les activités dans lesquelles notre économie n'est pas autosuffisante à I'heure actuelle.

L'objectif d'une reconquête du marché intérieur doit être d'accrốtre notre autonomie de décision tant en matière économique que politique, voire militaire. Sans nous fermer aux échanges avec l'extérieur, il convient donc de nous attaquer aux dépendances économiques qui hypothèquent le développement de notre économie et inhibent la liberté d'initiative de notre diplomatie. 


\section{Une priorité : la réduction de la dépendance énergétique}

Comme nous le verrons par la suite, la reconquête du marché intérieur passe par la mise en œuvre d'une stratégie globale visant à dynamiser l'économie française. En attendant que cette action de longue haleine porte ses fruits, des mesures d'urgence doivent être prises pour réduire dans les plus brefs délais notre contrainte extérieure. Dans cette optique, plusieurs arguments majeurs amènent à considérer la réduction de la dépendance énergétique comme tout à fait prioritaire.

1. L'énergie constitue de très loin le principal poste déficitaire de notre balance commerciale. En 1980 , les importations de produits énergétiques ont atteint 151,7 milliards de francs et le déficit commercial pour ces produits 132,9 milliards dont 111,7 milliards pour le pétrole brut. A titre de comparaison, la même année, le déficit commercial le plus important après l'énergie - celui de la filière bois regroupant des activités aussi variées que le papier et le carton, le meuble, le travail mécanique du bois, la sylviculture - n'a atteint «que » 13,1 milliards de francs. Toujours en 1980, les déficits commerciaux du matériel électronique ménager, d'une part, de l'informatique et de la bureaucratique, d'autre part, se sont élevés respectivement à 4,5 et 3,9 milliards de francs. Par conséquent, une réduction d'un même pourcentage, par exemple de $20 \%$, de notre dépendance en matière d'énergie et de notre dépendance en matériel électronique ménager aurait pour conséquence une diminution de notre déficit commercial de plus de 30 milliards de francs dans le premier cas et de moins de 1 milliard dans le second.

2. En dehors même de la responsabilité des importations d'énergie dans notre déficit commercial, celles-ci sont dangereuses à double titre : d'une part, en raison du rôle incomparable que joue l'énergie dans l'ensemble de la vie économique; d'autre part, en raison des incertitudes qui affectent non seulement l'évolution des prix de l'énergie, mais aussi les productions des principaux pays exportateurs dont la stabilité politique est pour le moins sujette à caution.

3. Si plusieurs secteurs méritent une attention particulière de l'Etat dans l'optique d'une reconquête du marché intérieur, seule l'énergie constitue une masse critique suffisante pour asseoir, au moins dans un premier temps, une relance économique fondée sur une telle reconquête du marché intérieur. 
4. Contrairement à ce qu'on pourrait penser à première vue, un programme de réduction accélérée de notre dépendance est sans nul doute beaucoup plus facile à mettre en ceuvre en matière d'énergie que dans tout autre secteur. En effet, l'énergie est un secteur centralisé qui dépend étroitement de l'Etat. Du reste, compte tenu de l'importance des investissements requis, aucune initiative de quelque ampleur ne paraît pouvoir être prise sans une impulsion décisive de celui-ci. En outre, les options énergétiques sont d'autant mieux adaptées à la planification que les choix qui s'offrent aux consommateurs en la matière se posent bien davantage en termes techniques et quantifiables qu'en termes subjectifs de goût : il ne suffit pas pour. reconquérir le marché de la chemise de produire en quantité suffisante des chemises solides et bon marché, il faut aussi et surtout que ces chemises satisfassent les goûts fluctuants des consommateurs, c'est dire que la marge d'incertitude est très grande; par contre, le choix entre différentes sources d'énergie, par exemple pour le chauffage, va dépendre essentiellement des prix comparés, des prestations techniques assurées (sécurité, etc.) quand ce n'est pas tout simplement des réglementations en vigueur. Or ces variables sont elles-mêmes largement déterminées par la politique énergétique adoptée.

Pour définir les actions de reconversion envisageables dans le prolongement du programme énergétique établi par le gouvernement et adopté par le Parlement durant l'automne 1981, il paraît judicieux de partir des diverses fonctions de l'énergie (chauffage des locaux, force motrice, etc.) plutôt que de raisonner en termes d'énergie primaire (pétrole, charbon, nucléaire, etc.). En effet, dans de nombreux cas, un besoin spécifique en énergie pourra être satisfait en remplaçant par exemple le pétrole par une énergie réunissant moins de qualités intrinsèques mais qui sera néanmoins parfaitement adaptée à ce cas précis.

La présentation d'un programme complet de reconversion énergétique dépasserait le cadre de cet article. Nous nous contenterons donc d'illustrer une nouvelle stratégie de réduction accélérée de notre dépendance énergétique avec un seul exemple : celui de la reconversion du chauffage des locaux et de l'eau chaude sanitaire ainsi que des usages basse température de l'industrie. Ce poste représente environ 35 à $39 \%$ de l'énergie importée.

Avec 100 calories de nucléaire, une centrale produit environ 30 calories d'électricité et rejette sous forme d'eau tiède 70 calories. Au prix d'une légère modification, parfois simplement d'un réglage différent, la centrale pourra produire 24 calories d'électricité mais les 


\section{Revue économique}

rejets thermiques vont être obtenus à une température beaucoup plus élevée, de l'ordre de 100 degrés, et seront alors utilisables pour alimenter des chauffages urbains. Du seul point de vue de la production d'électricité, le rendement de cette centrale électrocalogène va baisser 1. En revanche, si on prend en compte les deux productions - électricité et eau chaude - son rendement sera dorénavant de $100 \%$.

Quel sera le coût de ces nouvelles calories? Pour EDF, le manque à gagner sera de $30-24=6$ unités d'électricité. La société chargée de la commercialisation de l'eau chaude devra donc l'indemniser en conséquence. Moyennant quoi, elle obtiendra 76 calories d'eau chaude. Une calorie d'eau chaude devrait donc coûter à la sortie de la centrale 6/76 du prix d'une calorie d'électricité. Sachant que le kwh d'électricité nucléaire coûte environ 16,3 centimes, le coût du kwh thermique sera donc d'environ 1,29 centime. EDF demande un prix légèrèment supérieur en raison des aménagements qu'elle devra apporter aux centrales.

En tenant compte des autres coûts, inhérents notamment à l'installation des canalisations pour acheminer l'eau chaude vers les villes, on parvient à des prix de revient encore très avantageux. Par exemple, en Suède les autorités envisagent de remplacer l'alimentation actuelle en eau chaude de Stockholm obtenue à partir de plusieurs centrales combinées à combustible classique par une alimentation à partir d'une centrale nucléaire située à $120 \mathrm{~km}$ de la capitale. Le coût d'acheminement d'une quantité d'eau chaude équivalente à un kwh est estimé à 2 centimes environ. La centrale nucléaire de Dampierre, sur la Loire, se trouve à $130 \mathrm{~km}$ de Paris et pourrait donc alimenter Paris en eau chaude avec des coûts de transport comparables. Une estimation a été faite par une société de pose française en vue de l'alimentation de Strasbourg à partir de la centrale de Fessenheim située à $90 \mathrm{~km}$. Strasbourg ne compte que 300000 habitants. De ce fait, la puissance transportée sera plus faible et donc plus coûteuse que pour des villes plus importantes. Néanmoins, le coût n'a été chiffré qu'à 4 centimes par kwh.

D'autres coûts doivent cependant être pris en considération, notamment celui de la construction des réseaux d'eau chaude au sein même des villes. Ce coût est bien connu puisque des réseaux de chauffage urbain existent actuellement aussi bien en France qu'à l'étranger. Il se situe entre 3 et 4 centimes par kwh selon la taille des villes pour

1. Ces centrales électrocalogènes sont également intitulées : centrales mixtes, centrales combinées ou centrales à énergie totale. Le terme de cogénération s'emploie aussi de plus en plus. 
un investissement amortissable en dix ans. On peut également tenir compte des cô̂ts du stockage saisonnier : la chaleur peut en effet être stockée en été dans de grands réservoirs recouverts d'une couverture flottante en mousse de polyuréthane. De même, convient-il d'inclure le coût des calories de pointe produites localement avec des chaufferies ordinaires de manière à éviter de surdimensionner les canalisations à seule fin de satisfaire les consommations de pointe. En considérant l'ensemble de ces coûts, on arrivait avant les dernières hausses du prix du pétrole à un coût à la livraison aux consommateurs environ moitié moindre que celui du chauffage au fuel.

Au cours des prochaines années, les centrales vont rejeter davantage d'eau chaude que les villes ne pourraient en consommer. Selon le recensement précédent, la moitié des Français vivent dans des villes de plus de 50000 habitants. S'il est possible de relier les centrales à ces villes pour un coût non prohibitif, il paraît logique de reconvertir la plupart d'entre elles à cette forme de chauffage. Par ailleurs, $30 \%$ de la population vivent dans des villes ayant entre 2000 et 50000 habitants. Un grand nombre de ces villes petites et moyennes se trouvent sur le parcours que devraient emprunter. les canalisations entre les centrales et les grandes villes. C'est donc près de $80 \%$ des chauffages urbains qui pourraient être reconvertis.

Dans cette optique, en consacrant $10 \%$ du potentiel des entreprises du BTP à cette reconversion, un programme de l'ordre de 30 milliards de francs par an pourrait être lancé. Il permettrait d'obtenir une réduction de nos importations de pétrole de près de $40 \%$ en quatre à cinq ans ${ }^{2}$.

De manière plus générale, ce redéploiement énergétique pourrait s'inscrire dans une stratégie de réorientation de notre économie vers un développement plus autocentré ayant pour objectif non une spécialisation plus étroite, mais au contraire une meilleure adéquation de lappareil productif à l'évolution de la demande domestique.

2. Pour une étude plus détaillée de cette nouvelle politique énergétique, cf. Tovy Grjebine, Plan coordonné de reconversion énergétique pour la France, intergroupe "énergie ", Assemblée nationale. 
Au niveau des hommes comme à celui des entreprises, la politique suivie jusqu’à ces dernières années a toujours privilégié la formation d'une élite, quitte à accepter la croissance endémique de populations d'hommes et d'entreprises inadaptées à la société industrielle et a fortiori post-industrielle.

Sur le plan des hommes, le système éducatif élitiste conduit à une double impasse : d'une part, une large partie de la population qui a bénéficié d'une formation insuffisante pour lui permettre de remplir les tâches qualifiées que pourra offrir une économie en mutation; d'autre part, un «système de castes» qui empêche la diffusion des innovations, bloque le développement des formules originales, favorise le conservatisme économique et social.

Sur le plan des entreprises, la politique menée par MM. Giscard d'Estaing et Barre a privilégié un renforcement de la spécialisation de notre économie dans des secteurs de pointe considérés comme des «secteurs nobles» par opposition avec les secteurs traditionnels que l'on entendait abandonner progressivement aux pays à main-d'œuvre bon marché. Cette politique a eu deux conséquences particulièrement graves :

- un resserrement de la contrainte extérieure qui hypothèque notre développement. En 1980, les déficits commerciaux de secteurs traditionnels s'il en est comme ceux de la filière bois, du textile-habillement, du cuir-chaussure atteignaient respectivement 13,1 milliards de francs, 2,7 milliards et 2,1 milliards ;

- d'énormes gaspillages de fonds publics. En effet, même en considérant des secteurs tels que la sidérurgie ou le textile comme partiellement condamnés, le gouvernement ne pouvait accepter leur disparition rapide, compte tenu de l'importance de la main-d'œuvre employée dans ces secteurs $(568000$ personnes dans le textile-habillement en 1980, 256000 dans la sidérurgie, 110500 dans le cuir et la chaussure, etc.). Il a donc multiplié les subventions pour assurer la survie temporaire d'entreprises en difficulté et garantir la paix sociale plutôt que de permettre le développement de ces secteurs en favorisant leur modernisation.

La «société duale» apparaît comme l'aboutissement logique de ce double élitisme : au niveau des hommes, au niveau des entreprises. D'un côté, des entreprises ouvertes aux technologies nouvelles, compétitives à l'échelle internationale, employant une main-d'œuvre réduite mais hautement qualifiée. De l’autre côté, des entreprises tra- 
ditionnelles, incapables de faire face à la concurrence internationale sans protection et subventions, employant une main-d'œuvre abondante, peu qualifiée et mal payée. Le développement du chômage devait être pour ainsi dire le couronnement d'un tel système. L'expérience montre qu'une telle évolution n'est pas seulement catastrophique sur le plan social, elle l'est également d'un point de vue purement économique.

\section{Le paradoxe d'une politique industrielle réussie}

En réalité, il n'y a pas de secteurs dépassés, il n'y a que des techniques et des comportements obsolètes. Une économie dynamique ne repose pas sur quelques secteurs de pointe se détachant d'un tissu industriel amorphe mais sur une mutation permanente de l'ensemble des structures économiques et sociales. Le développement des nouvelles technologies doit donc être conçu de manière à favoriser non seulement l'éclosion d'activités nouvelles mais surtout la modernisation de tout l'appareil productif.

La réussite d'une politique industrielle repose sur le paradoxe suivant :

- lintervention de l'Etat se prête infiniment mieux au soutien du développement de technologies de pointe exigeant des investissements importants (nucléaire, téléphonie, télématique, etc.) qu’à une modernisation de l'ensemble du tissu économique et social ;

- mais la transformation d'éventuelles réussites techniques en succès pour l'ensemble de l'économie implique une large diffusion de ces technologies de pointe. Celles-ci doivent donc répondre à la demande d'un tissu économique et social dynamique en mutation permanente.

En déterminant ces options technologiques en fonction de leurs performances propres escomptées plutôt qu'en fonction des besoins fondamentaux de l'économie, les gouvernements ont, d'une part, privilégié les opérations de prestige sans lendemain dont le Concorde constitue le meilleur exemple, d'autre part, ils n'ont pas su ou voulu voir toutes les applications des nouvelles technologies développées. 


\section{Revue économique}

Par exemple, le nucléaire civil n’a été développé que pour produire de l'électricité alors qu'il aurait pu être à la base d'une reconversion énergétique plus large notamment par l'utilisation des rejets thermiques pour le chauffage urbain.

A contrario, si les efforts de $R$ et $D$ effectués ces dernières années, principalement au Japon et aux Etats-Unis, dans le domaine des semiconducteurs ont été sanctionnés par d'immenses succès commerciaux, c'est qu'ils allaient au devant d'une demande future domestique et mondiale très importante, émanant aussi bien des particuliers que des entreprises.

Les priorités sectorielles ne doivent donc pas être comprises comme un choix de secteurs d'avenir mais comme une sélection de secteurs critiques pour l'adaptation et la mutation de l'ensemble du tissu économique et social. La vocation universelle de ces secteurs permet de les regrouper sous le terme de «technologies génériques».

Ce constat conduit à distinguer d'une part les secteurs de technologies génériques qui nécessitent une intervention planifiée de l'Etat, d'autre part les autres secteurs caractérisés par une demande domestique et une pénétration étrangère fortes et/ou en croissance rapide qui appellent des actions plus souples de l'Etat.

\section{Une áction planifiée en faveur des technologies génériques}

Ces secteurs - qui évoluent à la mesure du progrès scientifique et technique - exigent une action planifiée de l'Etat pour quatre raisons majeures, les deux premières tenant à leur nature même, les deux autres à leur impact sur la vie économique :

1. Leur développement nécessite des investissements particulièrement importants, notamment en matière de recherche, qui dépassent souvent les capacités financières, technologiques et humaines des entreprises même de grande taille. A cet égard, il convient de préciser que nous n'avons en vue ici que les technologies nouvelles opérant une rupture radicale par rapport à des procès de production ou des produits existants. Elles ne doivent pas être confondues avec les innovations d'adaptation ou de démultiplication se situant dans le prolongement de technologies nouvelles et qui sont, dans bien des cas; réalisées pour un faible coût par des entreprises de moindre importance, voire par des PME. 
2. Il s'agit d'activité qui ne seront souvent rentables qu'à échéance plus ou moins lointaine. Seuls l'Etat ou le secteur nationalisé peuvent accepter de voir la rentabilité financière de leurs investissements ainsi différés.

3. La compétitivité - et donc finalement la pérennité - de l'ensemble des secteurs industriels plus traditionnels dépend de la diffusion de ces technologies.

4. Leur place dans la structure économique en fait des secteurs stratégiques pour lesquels la dépendance à l'égard de l'étränger équivaut à une perte d'autonomie politique et économique pour la nation.

Pour l'essentiel, ces technologies génériques appartiennent à un nombre réduit de secteurs : production d'énergie, informatique et bureautique, électronique, robotique et machine-outils, instruments et matériel de précision, biotechnologies auxquels on peut ajouter certaines technologies secrétées par la chimie, les industries spatiales, voire l'automobile et la mécanique.

L'arbitrage concernant le degré d'urgence de leur mise en œuvre pourrait résulter de la confrontation des critères suivants :

- importance du champ d'application (ou si l'on préfère : poids de la demande domestique actuelle et future);

- importance de leur effet d'entraînement sur les différentes branches de l'économie;

- importance du desserrement de la contrainte extérieure induit par le développement de chaque technologie (ou si l'on préfère : poids de la réduction du taux de pénétration);

- faiblesse de la demande d'importations exigées pour leur développement ;

- faiblesse du coût global de leur développement;

- faiblesse du risque politique qu'entraîne la dépendance vis-à-vis de l'extérieur pour chacune de ces technologies.

\section{Susciter l'adaptation permanente de l'ensemble du tissu économique et social}

Si une nouvelle politique industrielle doit porter en premier lieu sur le développement des technologies génériques, la diffusion de cellesci doit permettre la reconquête du marché intérieur dans des secteurs dont la demande domestique, d'une part, la pénétration étrangère, 


\section{Revue économique}

d'autre part, sont fortes et/ou en croissance rapide. On trouve dans cette catégorie tant des biens d'équipement spécialisés utilisés dans des secteurs particuliers que des biens de consommation modernes comme le matériel électronique ménager $(4,5$ milliards de déficit commercial en 1980) ou des secteurs traditionnels tels que ceux de la filière bois (papier-carton, meubles, etc.), le textile-habillement, le cuir-chaussure, le jouet, les instruments de musique, etc.

Plusieurs éléments rendent nécessaire le développement de ces secteurs :

- tout d'abord, nous l'avons vu, une économie dynamique ne peut reposer que sur un tissu économique et social aussi complet que possible s'adaptant en permanence et non sur quelques secteurs de pointe ;

- ensuite, le desserrement durable et profond de la contrainte extérieure passe par la reconquête du marché intérieur dans des secteurs qui jouent un rôle essentiel dans notre déficit commercial actuel. Ainsi, en 1980, le déficit cumulé d'un certain nombre de secteurs que l'on pouvait classer comme «traditionnels » atteignait 40 milliards de francs. Sans une action résolue des pouvoirs publics, cette situation risque de se détériorer rapidement et de nouveaux secteurs sont menacés (habillement, voire automobile, etc.);

- enfin, la mutation de notre économie exige lachat de brevets étrangers qui résultent de recherche excessivement coûteuses et qu'il serait sans doute vain d'entreprendre maintenant. Il parait donc judicieux de «réserver» les devises que nous acquérons en exportant pour financer de telles importations nécessaires au développement des technologies génériques plutôt que les «gaspiller» en achetant des biens de consommation qui peuvent, le plus souvent, être produits en France au prix d'une adaptation des secteurs concernés. A cet égard, il est intéressant de rappeler que, depuis la fin du siècle dernier, la politique japonaise a toujours suivi deux principes : 1 . éviter d'importer les produits qui ne sont pas indispensables à la croissance de l'industrie japonaise; 2. ne pas importer les produits qui sont ou peuvent être fabriqués au Japon.

En même temps, un développement planifié de ces secteurs paraît tout à. fait exclu pour trois raisons au moins :

- la multiplicité même des produits concernés conduirait une politique de reconquête du marché intérieur entreprise dans une telle 
optique à un gonflement demesuré de la planification dont on observe ailleurs les méfaits bureaucratiques;

- l'efficacité d'une politique de saupoudrage distribuant des subventions à un grand nombre de secteurs est éminement douteuse ;

- le développement des productions de biens de consommation se prête généralement mal à des interventions directes de l'Etat dans la mesure où dans ces secteurs la compétitivité dépend souvent de la rapidité de réaction aux variations de la demande et fait intervenir des éléments subjectifs comme la mode.

Il s'agit donc moins de lancer des plans de relance sectoriels que de créer les conditions nécessaires à une mutation permanente du tissu économique et social français :

- en améliorant la situation financière des entreprises par une politique de désendettement (cf. à ce sujet la troisième partie de cette étude) ;

- par un développement de la formation permanente qui devra être d'autant plus intensifié qu'il s'agit de remédier aux lacunes d'un système éducatif élitiste ;

- par une aide à la diffusion des technologies génériques. Celle-ci pourrait notamment prendre la forme d'un développement du leasing financé par l'Etat. Cette formule présenterait l'avantage considérable d'aider simultanément les producteurs nationaux (sans intervenir sur le front du commerce international) et les utilisateurs de ces technologies. Cette politique du leasing a servi de base au développement de l'industrie informatique japonaise et ce pays est aujourd'hui en train de mettre en cuvre ce même type de politique pour encourager le développement de la robotique;

- par la création d'un institut d'observation économique et industrielle comparable à ce qui existe au Japon qui serait chargé d'une double fonction : suivre les mutations de la demande et des techniques; contribuer à l'intégration des stratégies industrielles dans une vision macroéconomique du développement. 


\section{RENDRE A L'ETAT LE POUVOIR MONETAIRE}

L'orthodoxie budgétaire pratiquée avec plus ou moins de rigueur tout au long de la Cinquième République a eu pour conséquence de déposséder progressivement l'Etat de son pouvoir de création monétaire et de l'abandonner aux banques commerciales. Deux conséquences fondamentales en ont résulté :

- d'une part, la marge de manœuvre dont disposait l'Etat pour fixer les grandes orientations du développement économique a été réduite ;

- d'autre part, cette politique a préparé un terrain favorable à la stagflation. Pour le comprendre, il faut avoir à l'esprit que la monnaie peut être émise contre trois types de contreparties : des acquisitions d'or et de devises par la Banque de France, des avances au Trésor public ou des achats de bons du Trésor par l'Institut d'émission, enfin des crédits accordés aux entreprises et aux ménages par le secteur bancaire. Comme nul ne conteste la nécessité d'accroître la masse monétaire au fur et à mesure que la production nationale augmente, il est clair que la réduction d'une forme de création monétaire a inévitablement pour conséquence l'accroissement de l'une des deux autres. De ce fait, décider le maintien plus ou moins strict de l'équilibre budgétaire revient à accepter une création monétaire par un autre biais. C'est bien ce qui s'est passé, l'endettement des agents économiques se substituant peu à peu à celui de l'Etat. Cette mutation a eu pour conséquence une généralisation de l'intéressement à l'inflation. Les ménages endettés se contentent de bénéficier des remises de dettes que celle-ci leur assure. Les entreprises, elles, l'encouragent activement en représentant le coût des crédits qu'elles contractent sur leurs prix. Sur ce terrain propice, la montée des taux d'intérêt à partir de la fin des années 1960 a joué un rôle de détonateur mettant en mouvement le mécanisme de la stagflation.

Il s'agit maintenant de renverser cette évolution en rendant à l'Etat le pouvoir monétaire. La nationalisation du système bancaire ne saurait, à elle seule, y suffire. En effet, la lutte contre l'inflation exige que la création monétaire n'ait plus pour contrepartie un endettement des agents économiques, fût-ce à l'égard de banques commerciales nationalisées. 


\section{Changer les contre-parties de la monnaie}

Selon une idée solidement ancrée dans les esprit, la marge d'action dont dispose encore l'Etat pour s'attaquer à la contrainte extérieure est des plus étroites compte tenu des dépenses budgétairies déjà engagées ou prévues et des possibilités limitées de procéder à des emprunts publics sur le marché financier sans réduire d'autant les moyens disponibles pour le financement des entreprises. Cette idée repose sur une double confusion : entre une inflation par les coûts et une inflation par la demande; entre les différentes formes de création monétaire.

Tout d'abord, l'inflation actuelle ne pouvant être attribuée à un excès de la demande, une politique d'austérité monétaire, loin de favoriser une réduction durable de l'inflation, risque au contraire d'accentuer celle-ci. En fait, en limitant trop strictement la création monétaire, on ne peut que s'enfermer dans un cercle vicieux de la récession semblable à celui dans lequel la conjonction d'une politique monétaire restrictive et d'une politique de l'offre inefficace a placé les Etats-Unis. Pour éviter une telle issue, une création monétaire plus importante doit être acceptée sous réserve qu'elle ne s'accompagne pas d'un endettement accru des agents économiques.

En effet, une croissance identique de la masse monétaire peut être obtenue de plusieurs manières et une création monétaire peut se substituer à une autre sans modifier la quantité globale de monnaie en circulation. Au cours des vingt dernières années, l'endettement des agents économiques auprès des banques commerciales a joué un rôle de plus en plus important dans la création monétaire au point d'être à l'origine en décembre 1981 de $86 \%$ de celle-ci. Nous avons vu également que ce mode de création monétaire était générateur de stagflation en même temps qu'il réduisait la possibilité de l'Etat de financer des opérations d'intérêt général.

Il apparaît donc nécessaire de mettre un terme à cette évolution pernicieuse, d'abord en modifiant les modes de création de la masse monétaire, ensuite en remplaçant au moins partiellement la «monnaie endettée » actuellement en circulation par une «monnaie libre » de tout endettement des agents économiques.

Il convient de noter qu'un tel changement des contreparties de la masse monétaire n'est pas forcément lié à un plus grand engagement de l'Etat dans la vie économique. Il serait même parfaitement concevable d'associer une telle modification de la création monétaire à 


\section{Revue économique}

un désengagement de l'Etat. Il suffirait, pour ce faire, de mettre en ouvre le changement des contreparties par une simple réduction des impôts allant de pair avec une certaine restriction de la création de monnaie par les banques. Pour fixer les ordres de grandeurs, rappelons que les crédits bancaires accordés aux entreprises à des fins de trésorerie s'élevaient en décembre 1981 à environ 340 milliards de francs et que, dans le budget 1982, le montant escompté de l'impôt sur les sociétés est de 71 milliards de francs. Il serait donc envisageable de désendetter partiellement les entreprises en décrétant une suspension de l'impôt sur les sociétés pour une durée de trois à quatre ans. En supposant inchangée l'évolution des dépenses publiques, il résulterait de cette réduction des impôts un accroissement d'ampleur équivalente de la création de monnaie par l'Etat. Progressivement, une contraction de la masse monétaire créée par les banques commerciales pourrait être obtenue par une forte augmentation du taux des réserves obligatoires ou un resserrement de l'encadrement du crédit.

Cependant, il serait pour le moins regrettable de procéder à ce changement des contreparties de la masse monétaire sans en tirer profit pour financer un programme de grande envergure visant à la reconquête du marché intérieur. En fait, de manière quelque peu paradoxale, la situation déplorable dont il a hérité offre au gouvernement une formidable opportunité : aussi longtemps que la modification des contreparties ne sera pas terminée, l'Etat pourra procéder à des dépenses exceptionnelles à condition de réduire simultanément la création de monnaie par endettement des agents économiques.

\section{Privilégier l'investissement public productif}

Cette nouvelle création de monnaie au profit de l'Etat ne saurait toutefois être employée pour financer des dépenses improductives fussent-elles socialement justifiées sous peine de voir le déficit extérieur devenir rapidement incontrôlable.

Une confusion fréquemment faite consiste à juger indistinctement la création de monnaie par l'Etat, quel que soit l'usage d'un déficit budgétaire ainsi financé. Cette forme de création monétaire est généralement entachée de suspicion et opposée à la création de monnaie par le système bancáire au profit des entreprises qui bénéficient - à tort - d'un préjugé favorable. Cette vision des choses conduit à 
préconiser de nouvelles mesures d'austérité alors que la relance a à peine fait sentir ses effets et que la réduction de la contrainte extérieure n’a encore été qu'amorcée.

En réalité, une création de monnaie par l'Etat est de nature foncièrement différente selon qu'elle sert au financement de dépenses improductives ou au financement d'investissements productifs: En revanche, ce dernier financement s'apparente au financement monétaire d'investissements privés qui atteignait près de 300 milliards de francs en décembre 1981. La seule différence entre les deux - différence il est vrai tout à fait fondamentale - réside dans le fait que la création monétaire par l'Etat est exempte de tout endettement privé alors qu'au contraire la création de monnaie par les banques commerciales repose sur l'endettement des agents économiques et apparaît de ce fait comme une des sources de la stagflation.

Dans cette optique, on est amené à préconiser un plafonnement des dépenses courantes de l'Etat - et en premier lieu du budget social - au cours des prochaines années. Par contre, les dépenses publiques affectées au renforcement de l'appareil productif - en particulier à la réduction de la dépendance énergétique - devraient être notablement accrues.

\section{Une nouvelle présentation du budget}

Nous avons déjà souligné la distinction fondamentale qu'il convenait d'établir entre les dépenses courantes de l'Etat et les investissements publics productifs. On 'retrouve partiellement cette distinction dans la présentation des dépenses budgétaires. La loi de finances distingue, en effet, d'une part, les opérations à caractère temporaire qui comprennent principalement les prêts et avances consentis par le Trésor à des entreprises ou à des collectivités publiques, d'autre part, les opérations à caractère définitif. Parmi ces dernières, on distingue les dépenses ordinaires et les dépenses en capital dont près de la moitié est constituée par des dépenses d'équipement militaire. En revanche, cette distinction disparaît au niveau des recettes où seules des opérations à caractère temporaire retracées par des comptes d'affectation spéciale et les comptes spéciaux du Trésor sont présentés séparément.

Faute de reprendre la même distinction au niveau des recettes et à celui des dépenses, on en vient tantôt à financer des dépenses de caractère définitif par un recours à l'emprunt, tantôt au contraire à 


\section{Revue économique}

financer des investissements publics productifs par le prélèvement d'impôts. Conscient de cette anomalie, le Comité des experts présidé par Jacques Rueff avait suggéré en décembre 1958 de distinguer un budget des dépenses et recettes publiques nécessairement équilibré et un budget de prêts rentables financés par l'appel à l'épargne. Le volume de ce dernier budget devait dépendre de la situation du marché, le ministre des Finances pouvant réduire ou supprimer certaines opérations si le marché ne lui fournissait pas les moyens nécessaires. Cette proposition n'avait pas été retenue à l'époque.

Mais, surtout, cette absence de distinction entre des opérations qui répondent à des fonctions foncièrement différentes de l'Etat conduit à ignorer l'une des plus importantes de celles-ci : alimenter l'économie en monnaie. Faute de satisfaire cette fonction en assurant luimême une croissance de la masse monétaire proportionnelle à la croissance de l'économie, l'Etat a laissé le soin de la création monétaire aux banques commerciales avec tous les inconvénients qu'un tel état de fait comporte.

Nous sommes donc amené à proposer une nouvelle présentation du budget en trois parties :

- un budget fiscal de recettes et dépenses courantes. Ce budget serait obligatoirement équilibré. Il inclurait non seulement les dépenses courantes dans leur conception actuelle mais également les dépenses en capital non rentables d'un point de vue économique comme les dépenses d'équipement militaire;

- un budget bancaire financé par des emprunts publics et consacré à des prêts de l'Etat, à des entreprises ou à des collectivités publiques;

- un budget de croissance alimentant l'économie en monnaie libre de tout endettement et pouvant servir soit au financement d'opérations exceptionnelles, comme un programme de reconquête du marché intérieur par exemple, soit au financement d'une partie du premier budget. Dans cette dernière hypothèse, le prélèvement fiscal nécessaire au financement du budget fiscal pourrait être réduit.

Cette nouvelle présentation du budget permettra d'en finir une fois pour toutes avec les vieux poncifs - tel celui de la planche à billets - qui empoisonnent depuis tant de décennies le débat politique français. Le budget fiscal obligatoirement équilibré satisfera le bon sens populaire qui demande que l'Etat comme le particulier ne dépense pas plus qu'il ne perçoit sous forme de recettes. Le budget 
bancaire répondra aux règles d'une bonne gestion qui veut que les investissements rentables - publics ou privés - soient financés par le recours à l'épargne. Le budget de croissance permettra à l'Etat d'alimenter la vie économique en monnaie au fur et à mesure que la quantité de biens et de services à échanger augmente de la même façon qu'il construit de nouvelles routes pour faciliter le transport d'un nombre toujours plus grand de marchandises. On peut espérer qu'une telle présentation du budget permettra enfin aux Français de surmonter le traumatisme hérité de l'expérience de Law et des assignats.

\section{Les mesures complémentaires}

Au fur et à mesure que la relance fera sentir ses effets et que les capacités de production se rapprocheront du plein emploi, le gouvernement devra veiller à ce que cette nouvelle politique ne conduise pas à une création monétaire excessive, tout en poursuivant un programme de désendettement systématique des agents économiques. A cette fin, quatre mesures complémentaires devraient être envisagées :

1. En même temps que le rôle de l'Etat dans la création monétaire redeviendra prééminent, la création de monnaie par les banques commerciales devra être réduite. Pour ce faire, diverses mesures peuvent être appliquées, en particulier, une forte augmentation du taux des réserves obligatoires. Un processus inverse à celui suivi depuis le début des années 1960 sera ainsi engagé.

2. Afin de supprimer définitivement toute tentation de s'endetter en spéculant sur l'inflation pour ne rembourser qu'une partie des dettes contractées, la stratégie de désendettement pourrait aller de pair avec une indexation obligatoire de tous les crédits bancaires. Cette disposition devrait s'étendre à tous les prêts déjà obtenus, étant entendu que des remboursements anticipés seraient autorisés.

3. Les avantages actuellement accordés à l'endettement des entreprises au détriment du financement des investissements par augmentation du capital devraient être supprimés. Cette différence de traitement est particulièrement flagrante du point de vue fiscal. Comme le note fort justement le rapport Dautresme : "Le système fiscal actuel divise par deux, lorsqu'une entreprise est bénéficiaire, le coût des emprunts comparativement à celui des fonds propres, puisque les frais financiers ne sont pas inclus dans le bénéfice imposable; la loi du 


\section{Revue économique}

13 juillet 1978 a réduit cette différence en rendant déductibles à la base de l'impôt les dividendes rémunérant les actions nouvelles émises contre numéraire entre le $1^{\text {er }}$ janvier 1977 et le 31 décembre 1981, mais pour une durée limitée à sept ans [dix ans pour les actions à dividende prioritaire sans droit de vote], et pour autant que la rémunération servie ne dépasse pas 7,5\%. 3 》

Cette différence de traitement explique, pour une bonne part, le fait qu'en 1980 par exemple, les augmentations de capital n'ont représenté que moins du tiers du financement externe des entreprises.

La réforme de l'épargne soumise au Parlement en automne 1982 prévoit de porter cette durée à dix ans et de supprimer la limite de 7,5\% de la valeur des actions.

4. Enfin, il -serait souhaitable d'alléger les besoins de trésorerie des entreprises industrielles en réduisant les délais de paiements. A l'heure actuelle, on peut estimer que plus d'un tiers de l'endettement des entreprises s'explique par des besoins de trésorerie. On peut donc réduire la propension à s'endetter des entreprises en veillant à ce que la monnaie créée par l'Etat leur parvienne plus rapidement. Dans cette optique, les délais de paiements dont bénéficie actuellement la distribution devraient être notablement réduits. De même, l'Etat devrait-il exiger de ses trésoriers-payeurs qu'ils accélèrent leurs paiements et sanctionner plus énergiquement des retards qui menacent parfois la survie même de certaines entreprises et les contraignent à s'endetter.

Contrairement à ce qu'on pourrait penser à première vue, la limitation des crédits bancaires envisagés ici ne devrait pas pénaliser les entreprises qui ne bénéficieront pas des commandes publiques passées dans le cadre du budget de croissance.

D'abord, parce que les mesures de désendettement intéresseront toutes les entreprises et réduiront leurs besoins d'emprunts à des fins de trésorerie. Ces besoins deviendront également moindres en raison de l'abaissement des délais de paiements préconisés.

Ensuite, parce que la relance amorcée par l'investissement se diffusera dans l'ensemble de l'économie. Les entreprises pourront ainsi écouler une production plus importante et les bénéfices qu'elles feront,

3. Rapport de la Commission d'éturde présidée par $\mathbf{M}$. David Dautresme, Le développement et la protection de l'épargne, ministère de l'Economie et des Finances, avril 1982 , p. 250.

4. Pour une étude détaillée des différentes formules envisageables en vue de établir une égalité de traitement entre le financement de l'investissement par l'emprunt et par augmentation de capital, on se reportera au rapport mentionné cidessus, en particulier à son chapitre vr. 
de ce fait, diminueront leurs besoins d'endettement. De plus, le retour à l'expansion réduira les frais fixes des entreprises, ceux-ci pouvant être répartis sur une production plus importante. Le parallélisme que l'on observe généralement entre les difficultés croissantes des entreprises provoquées par la récession et l'endettement de celles-ci va jouer en faveur du désendettement dès lors que la relance fera sentir ses effets.

Enfin, parce que l'égalisation des avantages attribués au financement des investissements par l'endettement et par augmentation de capital limitera sensiblement le rôle de l'endettement dans le financement des investissements.

En fin de compte, on voit que la stratégie de la relance autocentrée permettra d'assainir la situation financière des entreprises françaises tout en dotant l'Etat, les collectivités locales et les entreprises publiques de moyens considérables. La reconquête du marché intérieur recevra, de ce fait, une impulsion sans doute décisive. La relance de l'économie pourra être d'autant plus forte et plus durable qu'elle contribuera ̀̀ atténuer progressivement les deux maux qui hypothèquent aujourd'hui notre développement : la contrainte extérieure et l'inflation.

\author{
ANDRÉ GRJEBINE \\ Attaché de Recherche \\ Fondation nationale \\ des sciences politiques \\ [CERI]
}


